\title{
Integrating Patients' Expectations into the Management of Their Depression: Report of a Symposium at the European College of Neuropsychopharmacology Congress
}

\author{
Koen Demyttenaere $\cdot$ Ellen Frank $\cdot$ David Castle $\cdot$ Elif Cindik-Herbrüggen
}

Received: June 11, 2019 / Published online: August 9, 2019

(C) The Author(s) 2019

\section{ABSTRACT}

A symposium held at the 31st European College of Neuropsychopharmacology congress in October 2018 in Barcelona, Spain discussed patients' expectations of treatment of their depression and how these can be integrated into patient management. Since treatment noncompliance is a major problem in patients suffering from depression, it is important to identify patients' expectations to improve treatment

Enhanced Digital Features To view enhanced digital features for this article go to https://doi.org/10.6084/ m9.figshare.8943449.

K. Demyttenaere $(\bowtie)$

University Psychiatric Centre, University of Leuven,

Campus Gasthuisberg, Louvain, Belgium

e-mail: koen.demyttenaere@uzleuven.be

E. Frank

University of Pittsburgh School of Medicine,

Pittsburgh, PA, USA

E. Frank

HealthRhythms, Inc., New York, USA

D. Castle

St. Vincent's Hospital Melbourne, Melbourne,

Australia

D. Castle

The University of Melbourne, Melbourne, Australia

E. Cindik-Herbrüggen

Neuro-Psychiatrisches Zentrum Riem, Munich, Germany compliance and in turn efficacy. Currently, there is no established protocol for choosing the right antidepressant therapy, and physicians need to tailor the choice based on the type of depression, its predominant symptoms, medical and psychiatric history of patients, and their previous response to, and adverse events with, treatment. Treatment strategies also need to be adapted to each patient's personality/persona and their personal beliefs, and patients need to be aware of the potential for drug-associated adverse events such as emotional blunting, sexual dysfunction and loss of functional outcomes, as the expectation of these events may limit their impact on treatment discontinuation. Also, placebo effects remain frequent with treatment, and there is currently no agreed method for predicting response to therapy. Of the available methods to determine treatment response, pharmacogenetic testing has limited value while functional imaging may be valuable, but is not practical in routine clinical practice. Online cognitive behavioural therapy (CBT) represents a new option in the clinical management of patients with depression, particularly for patients who may not be able to access direct interaction with a psychotherapist because of the severity of their condition, their geographic location or socioeconomic situation. Online CBT can act as an adjunct to drug treatment and face-to-face psychotherapy, rather than as the sole form of treatment to aid in identifying a patient's needs, thus meeting 
the treatment gap and improving compliance and efficacy.

Funding: Servier.

Keywords: Depression; deprexis ${ }^{\circledR}$; Online CBT; Online cognitive behavioural therapy; Patient expectations

\section{INTRODUCTION}

Poor treatment compliance is a major problem in patients with depression $[1,2]$ and leads to reduced therapeutic efficacy. It has been observed that more than $50 \%$ of patients receiving antidepressant therapy discontinue treatment within the first 3 months [3, 4], and $30 \%$ of patients treated with psychotherapy discontinue therapy after three sessions, with more than $50 \%$ doing so after the first six sessions [3].

Despite the current trend towards personalised medicine in many conditions, treatment of depression continues to focus on the brain and the effect of neurotransmitter changes on patient mood and function. In order to improve compliance and treatment efficacy in patients suffering from depression, the medical knowledge of doctors must be symmetrically matched with the beliefs, representations and expectations of patients [5]. Moreover, patients' perceptions of an interaction with their physician do not always align with the physician's perception of the same interaction, and this can affect the patient's adherence to antidepressant therapy [6].

A symposium entitled "Integrating patients' expectations into the management of their depression" was held on the 7th of October 2018 as part of the 31st European College of Neuropsychopharmacology (ECNP) Congress in Barcelona, Spain. The aim of this symposium was to describe the different techniques that can be used to identify patients' expectations during the management of their depression, the ways in which physicians can fulfil patients' needs during the therapeutic process and how online cognitive behavioural therapy (CBT) tools can be used to engage patients in their own self-management. This review summarises the presentations made at this symposium.

\section{Compliance with Ethics Guidelines}

This article is based on previously conducted studies and presentations and does not contain any studies with human participants or animals performed by any of the authors.

\section{IDENTIFYING PATIENTS' EXPECTATIONS FOR THE TREATMENT OF THEIR DEPRESSION}

Patients' satisfaction with treatment increases when they experience an improvement in their symptoms over time, and vice versa [7]. Despite the clear relationship between persistent symptoms and treatment satisfaction, treatment effectiveness is often not assessed in patients with depression. Reports suggest that as many as $80 \%$ of physicians do not use the available depression scales while treating patients for a number of reasons [8]. Firstly, some physicians may consider that the ratings partly reflect their own competence: lower scores indicate a better treatment outcome, which indicates that their treatment choice was a wise one, and vice versa. Secondly, some physicians may feel that scales developed in an academic setting do not accurately represent individual patients' experiences or expectations. Max Hamilton described using the same rating scales in different patients with depression as 'placing them on the Procrustean bed'—one size fits all [9]. Furthermore, patients differ in their treatment expectations, and items on the scale relevant to one patient may not necessarily be important to another.

In routine clinical practice, the list of symptoms discussed by the treating physician or the parameters included on different depression rating scales may not necessarily be the symptoms that are important to patients with depression. The patient's own illness representation and beliefs about causality, control/cure and consequences affect the type of information they need from their health providers [10], 
and their willingness to engage with and adhere to treatment [11]. A number of methods have been used to identify the needs of individual patients with depression.

\section{Patient Surveys}

Patient surveys can be an effective way to identify patients' needs before treatment initiation. Although not published in academic literature, some patient surveys revealed that patients were less concerned about dysphoria and more concerned about their functioning and sense of well-being during the management of their depression. However, since the 1990s, the evaluation of depression has been evolving and patients are now able to identify the symptoms of depression that concern them most, as indicated by the results of a patient survey conducted by the Depression and Bipolar Support Alliance (DBSA) in 2016 [12]. The DBSA survey invited patients with depression via their online newsletter, local chapters and social media pages. A total of 896 surveys were completed by 447 patients who reported a diagnosis of unipolar disorder and 449 who reported having bipolar disorder. The top-ranked items from this survey were not a sense of well-being or functioning, but the actual symptoms of depression (Table 1) [12]. Patients reported that anxiety relief was one of their key determinants of treatment effectiveness. Patients also endorsed different strategies that were helpful to them in the management of depression, with the most highly endorsed ones being wellness strategies, followed by conventional medications and psychotherapy (Fig. 1).

\section{Patient Focus Groups and Other Group Processes}

Patients' needs can also be identified by conducting patient focus and consensus groups. An example of this is the work of the James Lind Alliance (JLA) in the UK, which is involved in priority-setting partnerships for a range of disorders [13]. The JLA has identified research priorities in depression using a multiphase process that included patients, caregivers and
Table 1 Top-rated items during the Depression and Bipolar Support Alliance (DBSA) 2016 patient survey [12]

\begin{tabular}{ll}
\hline $\begin{array}{l}\text { How do you know if the } \\
\text { treatment is working? }\end{array}$ & $\begin{array}{l}\text { Patient response, } \\
\%(N=370)\end{array}$ \\
\hline $\begin{array}{l}\text { I don't feel overly anxious, agitated } \\
\text { or irritable }\end{array}$ & 62.7 \\
$\begin{array}{l}\text { My negative self-talk goes down } \\
\text { I don't dwell as much on negative }\end{array}$ & 54.4 \\
$\begin{array}{l}\text { experiences } \\
\text { I get out of bed in the morning/I } \\
\text { don't feel so tired or lethargic }\end{array}$ & 51.4 \\
$\begin{array}{l}\text { I am able to maintain concentration } \\
\text { for activities such as reading a }\end{array}$ & 44.6 \\
book & \\
\begin{tabular}{l} 
I feel hopeful about the future \\
\hline
\end{tabular}
\end{tabular}

healthcare professionals [14]. The process used online surveys to collect data from over 3000 respondents across the UK. The data were assessed to extract themes, which were then rated online by the respondents to identify priorities. In a subsequent workshop, survey respondents discussed the themes with the highest ratings to reach a consensus on the top 10 priorities for research in depression. Among these research priorities were several related to patients' expectations, including prevention of recurrence, early intervention both in the course of illness and course of an episode to reduce suffering, and psychoeducation that empowers patients in facilitated self-management. The outcomes of this consensus helped to identify the top priorities of patients with depression, their caregivers and the healthcare professionals who were directly involved in the management of depression.

\section{Qualitative Interviewing}

The Fondation FondaMental Expert Center's guide for patients (https://www.fondationfondamental.org/), which was designed using qualitative interviewing, emphasizes patients' expectations of their treatment for depression, 


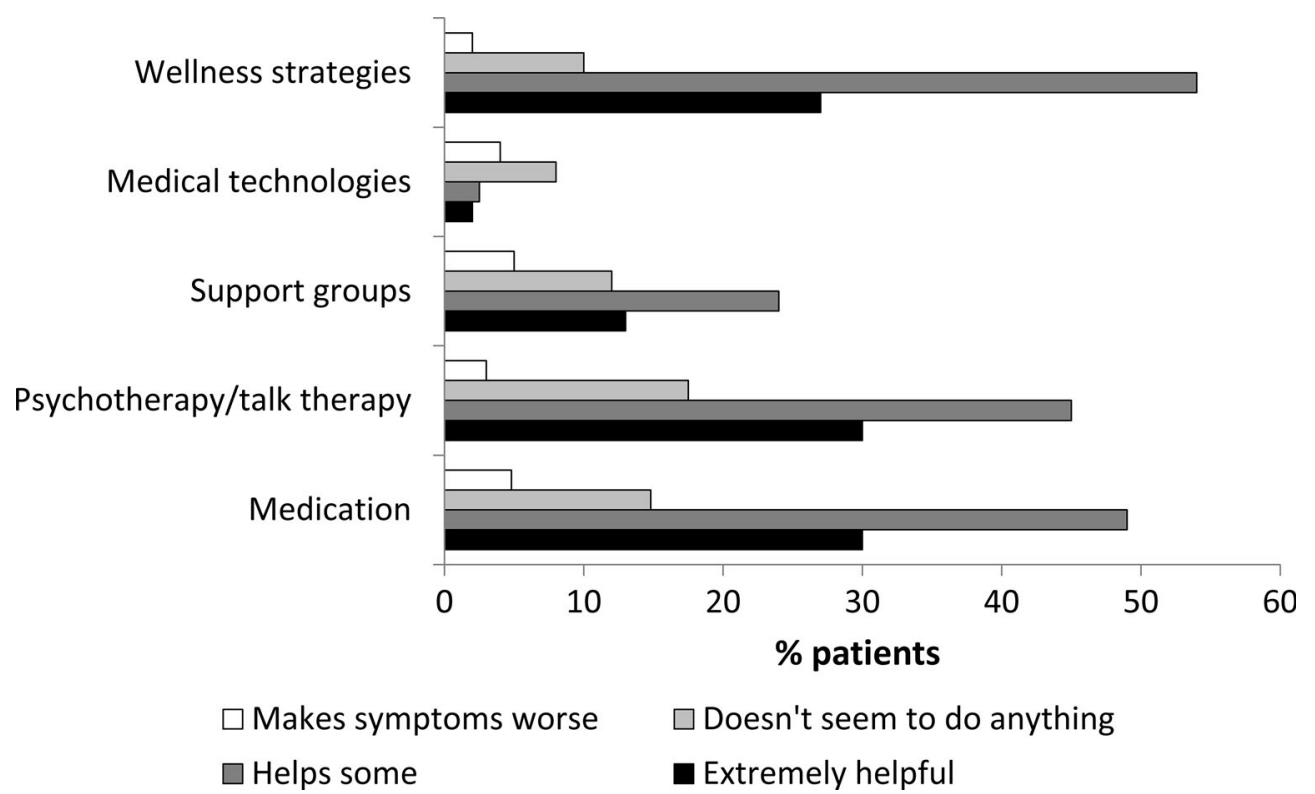

Fig. 1 Effectiveness of the treatment plan as per the Depression and Bipolar Support Alliance 2016 patient survey [12]

including active and collaborative involvement of patients in treatment selection, availability of sufficient information about current treatments to make an informed choice, and development of patient-specific treatment objectives. Qualitative interviews of patients with depression were used to develop this guide, which involved the following steps: (1) the centre directors questioned the staff members in a qualitative process and summarised their recommendations; (2) the recommendations were then incorporated into an informational resource for patients with depression and their families [15]. A key recommendation of this guide is to set treatment goals that are tailored to each patient, by specifically identifying how individual patients prioritize their symptoms, rather than simply following a standard protocol for the treatment of depression that does not consider the patient's priorities.

\section{Direct Questioning}

Direct questioning involves asking patients questions about the treatment received. This method was used by the Depression and Manic Depression Prevention Program (DMDPP) at the
University of Pittsburgh, PA, USA to conduct trials focussed on the long-term maintenance of wellness in patients with depression and bipolar disorders [16-19]. At the end of the study, patients were questioned about what they found helpful during treatment. The patient responses highlighted the importance of respecting their belief systems ("My therapist prayed with me"), an egalitarian stance ("My doctor laughed at my jokes") and ensuring that patients felt that their appointments were not rushed ("My doctor gave me all the time in the world"). Similar results have been reported in research from the UK, in which direct questioning of patients with depression highlighted the importance of a trusting and positive therapeutic relationship between physician and patient, and a holistic patient-centred approach to care in which all of the patient's health concerns were considered, and not simply their mental health needs [20].

\section{Collaborative Design of Treatment- Related Technology}

The University of Pittsburgh, PA, USA has designed a smartphone-based application (app) 
for patients with mood and anxiety disorders called CUE. This app uses sensors on the phone to automatically measure patients' daily behaviour including mood and energy, social interaction, activity and sleep/awake regulation. CUE can identify any change in patients' behaviour based on the recorded data and provides an update on their weekly behavioural profile, which may be useful for identifying methods to improve their symptoms. For example, if the data indicate that the patient is spending too much time at home, the healthcare provider may suggest going out for a walk with a friend. CUE also provides psychoeducational learning units for patients.

CUE was beta-tested in patients with depression and anxiety, who downloaded the app and used it for 28 days (unpublished data). Semi-structured interviews were conducted by mental health professionals on days 3, 14 and 28 , focussing mainly on usability, what the patients liked and disliked about the app, and any functionality or items that were missing. Patients who used CUE during the testing responded positively, stating that using CUE was like having a companion and reduced the feeling of loneliness, and helped them to transition from resisting their diagnosis and treatment to accepting and embracing the process. However, patients wanted more psychoeducational learning units, in-depth psychoeducational material, feedback about their own behaviour and feedback about how they were changing (or not). Development and refinement of the CUE app is ongoing.

Overall, patients with depression want relief of their symptoms, a sense of well-being and improved functioning, early intervention and prevention of recurrence with treatment. In order to achieve these goals, patients also want a collaborative and egalitarian relationship with their treating clinician, along with in-depth information about both their disorder and effective treatment interventions. Although there is no empirical literature available to understand patients' expectations, different available methods can be used to obtain this information.

\section{CHOOSING THE RIGHT ANTIDEPRESSANT REGIMEN}

Although pharmacotherapy remains the main treatment strategy for depression, there are no accepted protocols for selecting the right treatment that can effectively treat the symptoms, be well tolerated and, most importantly, be adhered to by the patient. In addition, current methods for predicting treatment response have limited value in routine clinical practice.

In an attempt to compare the efficacy and safety of different antidepressants in the management of depression, the STAR*D trial studied a pre-set strategy for the management of depression, in which patients were treated with different antidepressants in a stepwise manner $[21,22]$. Step 1 consisted of treatment with citalopram monotherapy, which showed a modest remission rate (RR) of $36.8 \%$. In step 2 , patients chose one of four options: switch to venlafaxine/bupropion/sertraline, combine citalopram with bupropion/buspirone, switch to cognitive therapy or combine citalopram with cognitive therapy, with an RR of $30.6 \%$. Patients who further switched to nortriptyline/ mirtazapine or augmented antidepressant therapy with lithium/triiodothyronine in step 3 showed an RR of $13.7 \%$, and finally switching to tranylcypromine/mirtazapine + venlafaxine in step 4 showed an RR of 13\% [21]. These results were somewhat disappointing, and many psychiatrists felt that they could achieve better outcomes in clinical practice by using informed clinical judgement versus the prescribed stepwise approach.

Given the current lack of defined protocols for antidepressant drug selection, clinicians usually select an appropriate treatment on the basis of the patient's clinical and social context and their previous treatment history, and individualise treatment with the aim of restoring their function. For newly diagnosed patients, treatment choice should consider their family history of depression, if any, the medications used and their responses to the treatments. The medication selected should be effective for the symptoms reported by patients and be well tolerated. In order to further individualise 
treatment, patients should be advised of the potential for any treatment-associated adverse events (AEs; e.g. agitation, akathisia, sexual dysfunction, weight gain, etc.) so that they (the patient) can identify any AE that they are unlikely to tolerate. For example, patients with body image disorders may be reluctant to take antidepressants that cause long-term weight gain. Patients should also be advised that there may be a delay between treatment initiation and symptomatic improvement, and that some depressive symptoms may temporarily worsen in the first weeks of therapy, because this information can help patients to persist with treatment during the early weeks.

The choice of medication should also be based on the type of illness (bipolar, mixed states, psychotic, melancholic, atypical, etc.) and their individual symptoms. In clinical practice, patients with depression often show a mixture of symptoms that may indicate other types of illness (called 'missed mixed state'), as demonstrated in the Bipolar Disorders: Improving Diagnosis, Guidance and Education (BRIDGE) study [23], which found that up to $47 \%$ of patients with major depression enrolled in the study also had bipolar disorder. Therefore, correct diagnosis of mixed symptoms is required before treatment selection to avoid worsening of symptoms.

\section{Predicting Patients' Response to Antidepressants}

In an attempt to determine the usefulness of pharmacogenetics in psychiatric treatments, Zubenko et al. reviewed the available literature and found only ten relevant published studies with a modest number of patients $(n=3219)$ [24]. The studies largely focused on markers of metabolism for antidepressant agents and did not meet the criteria for high-level evidence. Owing to the limited literature available on the clinical efficacy of pharmacogenetic-guided therapy, the authors conclude that antidepressants need to be dosed by careful choice, and monitoring of therapeutic effects and AEs, rather than solely on pharmacogenetic tests [24].
The role of functional imaging in predicting treatment-specific response in treatment-naïve patients with depression was studied by Dunlop et al. in the Prediction of Remission to Individual and Combined Treatments (PReDICT) study [25]. Patients were randomised to receive CBT or antidepressants (escitalopram/duloxetine) for 12 weeks. Functional connectivity of the subcallosal cingulate (SCC) was measured using magnetic resonance imaging (MRI) before randomisation. Of the 122 patients who had MRI data available, 58 showed remission and 24 showed treatment failure after 12 weeks of treatment. The sum of the baseline functional connectivity scores of the SCC was positive in patients who achieved remission with CBT and treatment failure with medication, and negative in patients who showed remission with medication and treatment failure with CBT [25]. These results suggest that functional imaging may help to predict the outcomes of first-line treatment of depression, although more studies are needed to confirm this. Also, it may not be possible for all patients to undergo a functional MRI before treatment in routine clinical practice for various reasons, including availability and cost. While it is important for clinicians to have up-to-date knowledge of available techniques in the field, they should try to integrate these techniques into clinical practice, when possible, to achieve maximum benefit.

\section{Attitudes, Expectations and Placebo Effects}

In patients with depression, expectancy bias (the belief that a treatment will or will not be effective) may affect assessments of drug efficacy in clinical practice as well as in clinical trials. This effect was clearly seen in a prospective randomised controlled trial where patients with major depressive disorder (MDD) were first randomised to receive 8 weeks of treatment with open-label citalopram or placebo, and the placebo recipients were then re-randomised to blinded treatment with citalopram or placebo [26]. The patients who received open-label citalopram showed significantly higher expectancy ratings $(12.1 \pm 2.1$ vs $11.0 \pm 2.0$; 
$p=0.03)$ compared with those who received placebo and entered the blinded phase, and had more rapid reductions in depression ratings with treatment. These results showed that patients' expectations partially mediated the antidepressant effects of treatment. Linden called this a 'nocebo effect' and suggested several ways of limiting its impact [27], including reducing patient uncertainty regarding the efficacy of treatment ("Even if the therapist feels unsure about the prognosis, it is reasonable to tell the patient that this is the best treatment available"); conveying an optimistic and hopeful attitude ("Tell the patient that the treatment will work and the future will be fine"); encouraging the patient to observe positive responses to interventions ("whether they are treatmentrelated or not"); and conveying optimism by 'suggestion' ("Tell the patient how he or she should feel"). He further stated that "It is in any case ethical to say that therapists should avoid nocebo and foster placebo effects" [27].

The patient's treatment preference does not appear to affect treatment efficacy, but can impact adherence. In the PReDICT study, patients did not show any particular preference for treatment, with approximately one-third of patients having no preference, one-third preferring medications and one-third preferring CBT [28]. Furthermore, the HAM-D scores (11.1 vs 11.2 vs 10.2$)$ and the RR ( $46.7 \%$ vs $54.7 \%$ vs $41.9 \%$ did not significantly differ between patients treated with escitalopram, duloxetine or CBT during the study. However, among patients who expressed a treatment preference, treatment completion rates were higher in those who received their preferred treatment versus those who did not $(82 \%$ vs $68 \%$; $p=0.013$ ) [28].

The relationship between a patient with depression and their treating physician should be bidirectional. Physicians need to provide patients with appropriate information on depression and the available treatment options, and understand patients' expectations so that the patient can participate in the decisionmaking, which in turn can improve treatment compliance and efficacy (Table 2) [29].

\section{Potential Impact of Adverse Events on Treatment Choices}

\section{Emotional Blunting}

Emotional blunting is common with most antidepressants, especially the selective serotonin reuptake inhibitors (SSRIs) [30, 31]. Patients reporting their response to antidepressants on internet forums invariably describe emotional blunting in negative terms, for example: "I don't feel particularly depressed, but have completely withdrawn and emotionally cut off from everything", "I do not wish to live in this permanently deadened, apathetic state" and "I am not willing to continue with the flat affect". Qualitative interviews of patients on SSRI therapy have also reported general effects on all emotions, reduction of positive and negative emotions, emotional detachment, indifference, effects on everyday life (helpful/not helpful), alterations in personality and blunting associated with SSRIs [32].

Emotional blunting may not be seen with all antidepressants. Agomelatine, a melatonin agonist and $5-\mathrm{HT}_{2}$ antagonist, has been reported to cause less marked emotional blunting compared with SSRIs. A double-blind controlled study conducted in patients $(n=324)$ randomised to either agomelatine $(25-50 \mathrm{mg} /$ day) or escitalopram (10-20 mg/day) for 24 weeks reported that although the two treatments showed similar efficacy in improving the symptoms of depression (change from baseline in the Hamilton Depression Rating Scale [HAM]-D scores - 19.9 with agomelatine vs - 19.2 with escitalopram), a higher proportion of patients in the escitalopram group felt that things they cared about before the illness did not seem important anymore compared with agomelatine $(53 \%$ vs $16 \% ; p=0.024)$ [33]. Similarly, another open-label parallel-group study in patients with MDD who were randomised to receive agomelatine $(25-50 \mathrm{mg} /$ day; $n=30)$ or venlafaxine $(75-150 \mathrm{mg} /$ day; $n=30)$ for 8 weeks showed that although both treatments significantly reduced the HAM-D, Hamilton Anxiety Rating Scale (HAM-A) and the Snaith Hamilton Rating Scale (SHAPS) scores, the reduction from baseline in the SHAPS score at week 8 was significantly greater 
Table 2 Characteristics of shared decision-making. Adapted from Hamann et al. [29]

\begin{tabular}{|c|c|c|c|}
\hline & $\begin{array}{l}\text { 'Traditional medical model' } \\
\text { (paternalistic model) }\end{array}$ & Shared decision-making & Informed choice \\
\hline $\begin{array}{l}\text { Role of doctor } \\
\text { (active) }\end{array}$ & $\begin{array}{l}\text { Active: reports only selected information to } \\
\text { the patient, chooses the therapy he } \\
\text { considers best for the patient }\end{array}$ & $\begin{array}{l}\text { Active: reports all } \\
\text { information and } \\
\text { treatment possibilities to } \\
\text { the patient } \\
\text { Can recommend an option } \\
\text { Decides on the therapy } \\
\text { together with the patient }\end{array}$ & $\begin{array}{l}\text { Passive: reports all } \\
\text { information and treatment } \\
\text { possibilities to the patient } \\
\text { Withholds his } \\
\text { recommendations } \\
\text { Makes no decision }\end{array}$ \\
\hline Role of patient & $\begin{array}{l}\text { Passive: accepts the proposal of the doctor. } \\
\text { Is obliged to cooperate in his recovery }\end{array}$ & $\begin{array}{l}\text { Active: receives all } \\
\text { information } \\
\text { Forms his own judgment on } \\
\text { harms and benefits of } \\
\text { treatment options } \\
\text { Discusses his preferences } \\
\text { with the doctor } \\
\text { Decides on the therapy } \\
\text { together with the doctor }\end{array}$ & $\begin{array}{l}\text { Active: receives all } \\
\text { information } \\
\text { Forms his own judgment } \\
\text { Is free to choose between all } \\
\text { options unbiased by his } \\
\text { physician's opinion } \\
\text { Decides on the therapy alone }\end{array}$ \\
\hline $\begin{array}{l}\text { Information } \\
\text { flow }\end{array}$ & One way: patient $\rightarrow$ physician & $\begin{array}{l}\text { Bidirectional: } \\
\text { patient } \leftrightarrow \text { physician }\end{array}$ & $\begin{array}{l}\text { One way: patient } \leftarrow \\
\text { physician }\end{array}$ \\
\hline $\begin{array}{l}\text { Responsibility } \\
\text { for the } \\
\text { decision }\end{array}$ & $\begin{array}{l}\text { Lies with the doctor } \\
\text { High risk of being sued for wrong decisions }\end{array}$ & $\begin{array}{l}\text { Lies with both the doctor } \\
\text { and patient }\end{array}$ & $\begin{array}{l}\text { Lies with the patient } \\
\text { Low risk of the physician } \\
\text { being sued for wrong } \\
\text { decisions }\end{array}$ \\
\hline
\end{tabular}

with agomelatine compared with venlafaxine $(p<0.01$; Fig. 2) [34]. However, this was a pilot study, and these results will need to be confirmed in a larger patient population.

Emotional blunting can be measured using different scales, including the Castle Hopwood Emotional Experience Response (CHEER) index. The CHEER index was developed with the specific objective of reminding psychotherapists to ask patients about their general emotional state in order to identify blunting (Table 3 ). The scale rates a patient's emotional state on the basis of their response to simple questions about both sad and positive events [35]. The CHEER index was used to determine emotional blunting in general practice in the PRISM study $(n=6576)$, which reported low blunting in $13 \%$ of patients, moderate in $41 \%$, and high in $46 \%$ of patients; these results need to be confirmed [35].

Demyttenaere et al. developed another scale for measuring emotional blunting in patients with depression called the Leuven Affect and Pleasure Scale (LAPS) [36]. The LAPS is a 16-item scale in which treated patients rate their positive and negative moods, and hedonic tone (Table 4) from 0 to 10 , where 0 is not at all, $1-3$ are scores for a little bit, 4-6 are scores for moderately, 7-9 are scores for quite a bit and 10 represents very much. The LAPS was confirmed 


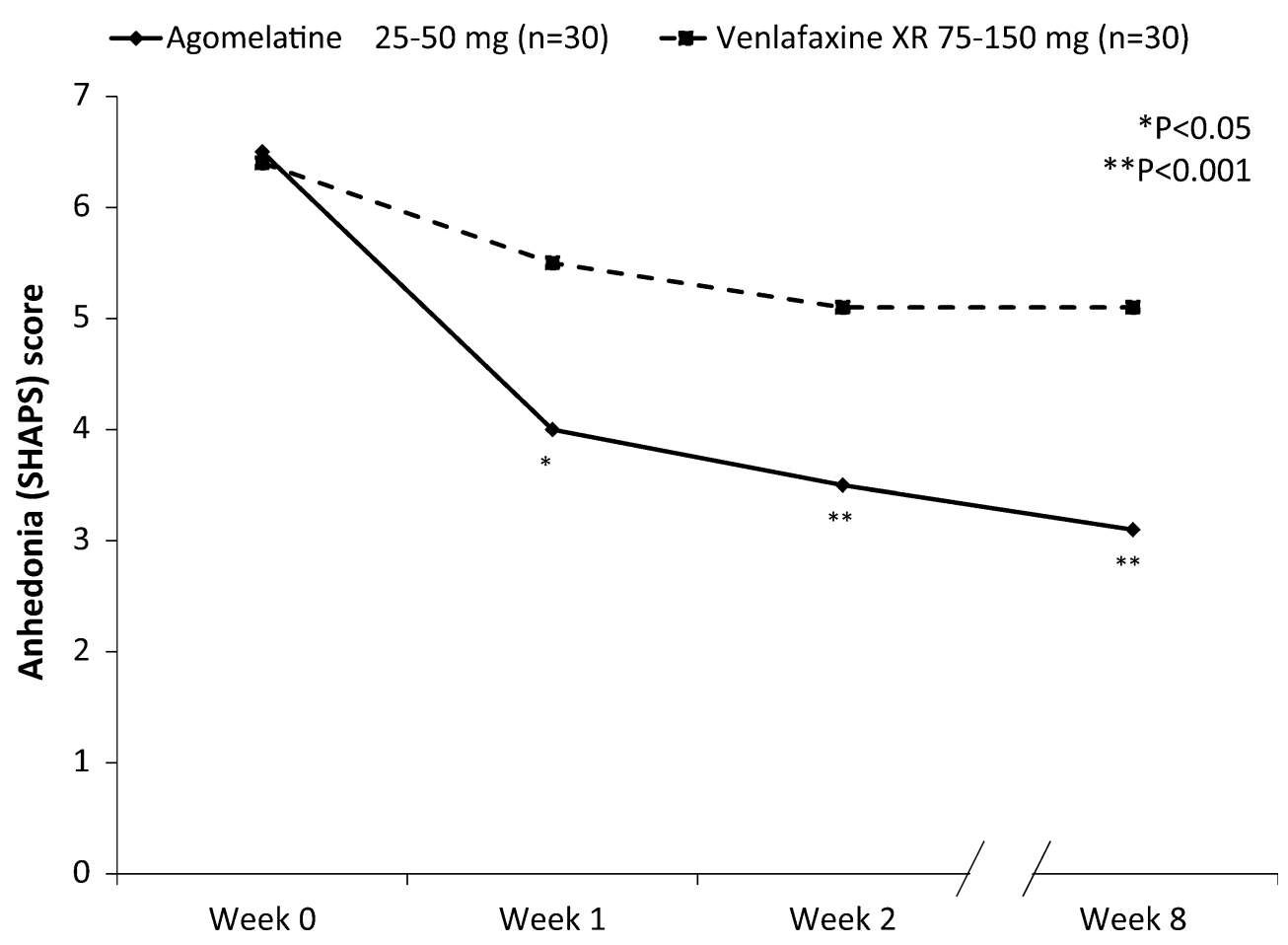

Fig. 2 Change from baseline in the Snaith Hamilton Rating Scale (SHAPS) scores for agomelatine versus venlafaxine during the 8-week study. Reproduced with permission from Martinotti et al. [34]

in college students who were healthy $(n=138)$, depressed but functioning $(n=27)$ or severely depressed $(n=38)$ [36]. The results showed moderate correlation between the positive and negative factors, and the independent validators (cognitive functioning, overall functioning, meaningful life, happiness) were strongly correlated with positive affective factors [36].

\section{Sexual Dysfunction}

Sexual dysfunction is another common AE with antidepressant treatment, and most patients with depression experience ongoing sexual dysfunction. It is therefore important for clinicians to appropriately identify any sexual difficulties experienced by the patient before initiating treatment in order to choose a medication that does not worsen their existing symptoms. Exacerbation of sexual dysfunction with treatment may lead to early discontinuation of treatment. A meta-analysis of treatment-emergent sexual dysfunction in patients with depression reported a higher rate of sexual dysfunction with SSRIs and serotonin-norepinephrine reuptake inhibitors (SNRIs) compared with other available antidepressants [37]. However, agomelatine was one of the few agents associated with no sexual dysfunction and was the only drug with an incidence of sexual dysfunction significantly lower than that of placebo (Fig. 3) [37]. These results indicate that agomelatine may be a suitable treatment option in this patient population, although larger trials are required to confirm this effect.

\section{INVOLVING PATIENTS \\ IN THE MANAGEMENT OF THEIR DEPRESSION: ROLE OF E-CBT}

Early diagnosis and treatment of depression is essential to prevent recurrent episodes. The German national S3 treatment guidelines for unipolar depression consider psychotherapy and pharmacotherapy as equally effective and valuable options in the management of depression [38]. However, patients with severe 
Table 3 Elements on the Castle Hopwood Emotional Experience Response (CHEER) index scale [35]

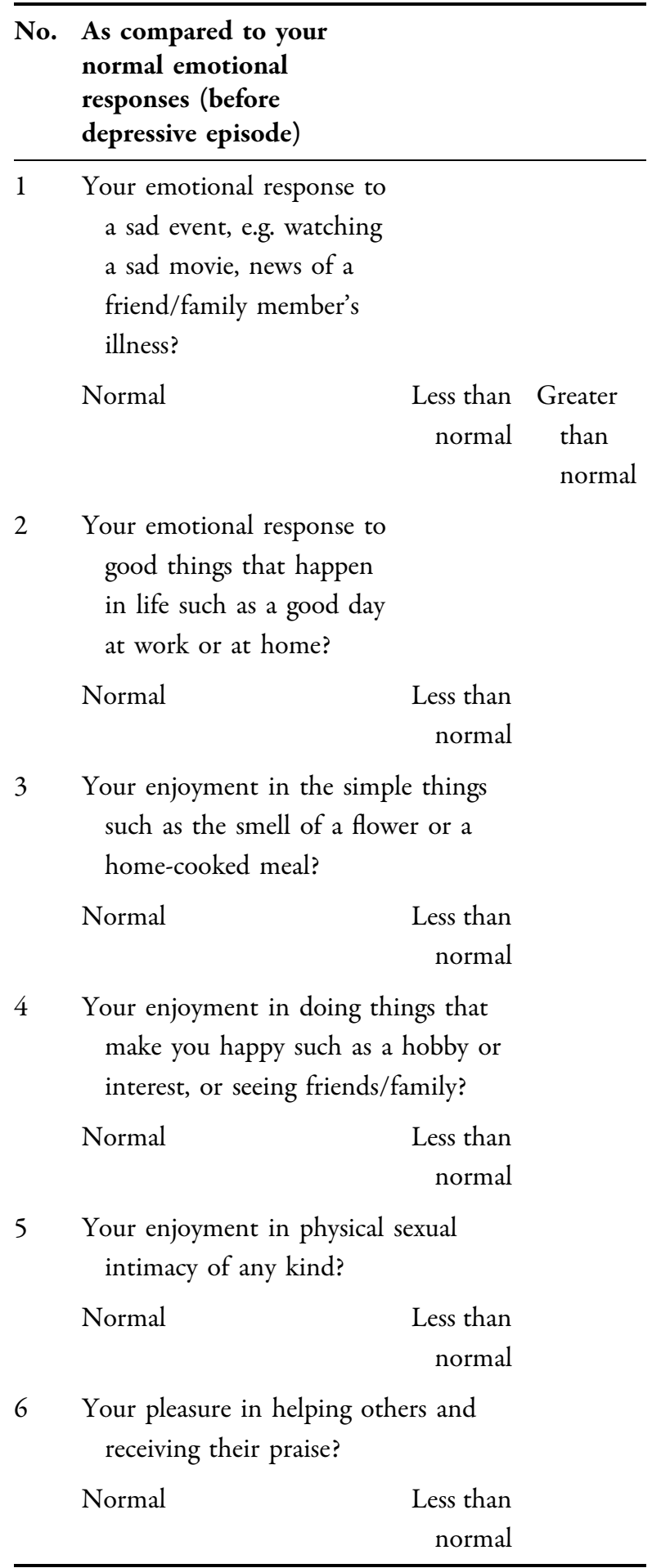

depression and cognitive impairment may not benefit from psychotherapy alone, and need to receive appropriate medications before psychotherapy is offered. Early diagnosis and treatment of depression may not always be possible, particularly in areas with limited access to specialist psychiatrists or other diagnostic or therapeutic services. For example, in Germany, the average waiting time to see a psychiatrist is 13 weeks [39], and the average time to initiate psychotherapy is between 6 and 12 months [40]. Immigrants who do not speak German may have extended waiting times of up to 24 months. It is therefore important to look for alternatives that can fill this gap.

\section{Online Cognitive Behavioural Therapy: deprexis $^{\circledR}$}

The deprexis ${ }^{\circledR}$ interactive online program is available in Germany for patients with unipolar depression or depressed mood. It utilises recognised methods and techniques of CBT and is designed to be used alone or in combination with pharmacotherapy/psychotherapy in the management of depression. The deprexis ${ }^{\circledR}$ program provides information on the clinical details of depression along with exercises and techniques for depression management. The program does not require special computer skills, and supports patients by sending email and text reminders to encourage them to undertake these exercises daily.

The deprexis ${ }^{\circledR}$ program can be used effectively by patients with mild to moderate depression who are willing to use this online intervention. It consists of 11 content modules (Table 5), of which nine represent psychotherapeutic approaches. Each module can be completed within $60 \mathrm{~min}$, depending on the user's reading speed, interest, motivation and individual path through the program. Modules are organised as simulated dialogues in which the program explains and illustrates concepts and techniques, engages the user in exercises and continuously asks users to respond by selecting from response options. Subsequent content is then tailored to the user's responses, resulting in a simulated conversational flow. All modules 
Table 4 Items in the Leuven Affect and Pleasure Scale (LAPS) [36]

On a scale of 0 to 10 , to what extent did you experience this in the past week?

Sad, depressed

Ashamed, guilty

Anxious, tense, nervous, stressed, afraid, scared, jittery

Irritable, critical, angry, hostile, frustrated, being upset

Energetic, lively, strong, interested, inspired, joyful, enthusiastic, proud

Safe, trustful, understood, warmly surrounded

Calm, content, zen, peaceful, balanced, relaxed, serene

Loving, friendly, caring, compassionate, grateful

Having interest in or looking forward to a good meal or a drink, touching and being touched, a warm and sunny day, smelling the forest or the sea, listening to music, walking on the beach, looking at something beautiful...

Taking pleasure from or enjoying a good meal or a drink, touching and being touched, a warm and sunny day, smelling the forest or the sea, listening to music, walking on the beach, looking at something beautiful...

Having interesting or looking forward to contacts with people important to me

Taking pleasure from or enjoying contacts with people important to me

I can think clearly, I can focus well. I can make decisions and my memory is good

I can function well (occupation, social and family life)

I feel my life is meaningful

I feel happy

are accompanied by illustrations (e.g. drawings, photographs, Flash animations).

Under the commercial name deprexis ${ }^{\circledR}$, the program has been validated in Germany [41] and was awarded the Emanuela Dalla Vecchia Prize of the German Society for Psychiatry and Psychotherapy, Psychosomatics and Neurology. The effectiveness of deprexis ${ }^{\circledR}$ for relieving depressive symptoms has been demonstrated in a number of randomised comparative studies of 8-12 weeks' duration [42-48], as well as in several meta-analyses $[49,50]$, with the most recent one reporting a medium effect size ( $g=0.51,95 \%$ CI $0.40-0.62)$ and low heterogeneity $\left(I^{2}=26 \%\right)$ [49]. In the randomised studies, adding deprexis ${ }^{\circledR}$ to usual care significantly improved a range of outcomes compared with usual care alone; these outcomes included symptoms of depression measured using various rating scales, quality of life, the Rosenberg SelfEsteem Scale and Dysfunctional Attitude Scale [41, 45-47]. Preliminary data from its use in German patients suggest that deprexis ${ }^{\circledR}$ plus usual care may also reduce the cost of care (in terms of statutory health insurance costs) relative to usual care alone [51], but further research is needed to establish the cost-effectiveness of deprexis ${ }^{\circledR}$ in other countries.

\section{Benefits of deprexis ${ }^{\circledR}$}

The use of online programs such as deprexis ${ }^{\circledR}$ can have a number of advantages. These programs can help to reduce waiting times for psychotherapy and provide patients with the basic principles of depression management before therapy can be initiated. They also offer an alternative platform for patients to open up, which can be particularly useful for patients who struggle to speak to their therapist, and provide an additional therapeutic pathway to augment the limited number of sessions a patient has with their psychiatrist. Being an online intervention, deprexis ${ }^{\circledR}$ allows access to therapy for patients in rural areas, those with restricted mobility or patients who have limited time for the therapist because of excessive workloads, helping patients to maintain their ability to work while receiving treatment.

\section{Using deprexis ${ }^{\circledR}$ in Clinical Practice}

Not all patients with depression may be comfortable with internet-based interventions. Some patients fear that the insurance company will access their symptoms from the online database, which may impact their employment 


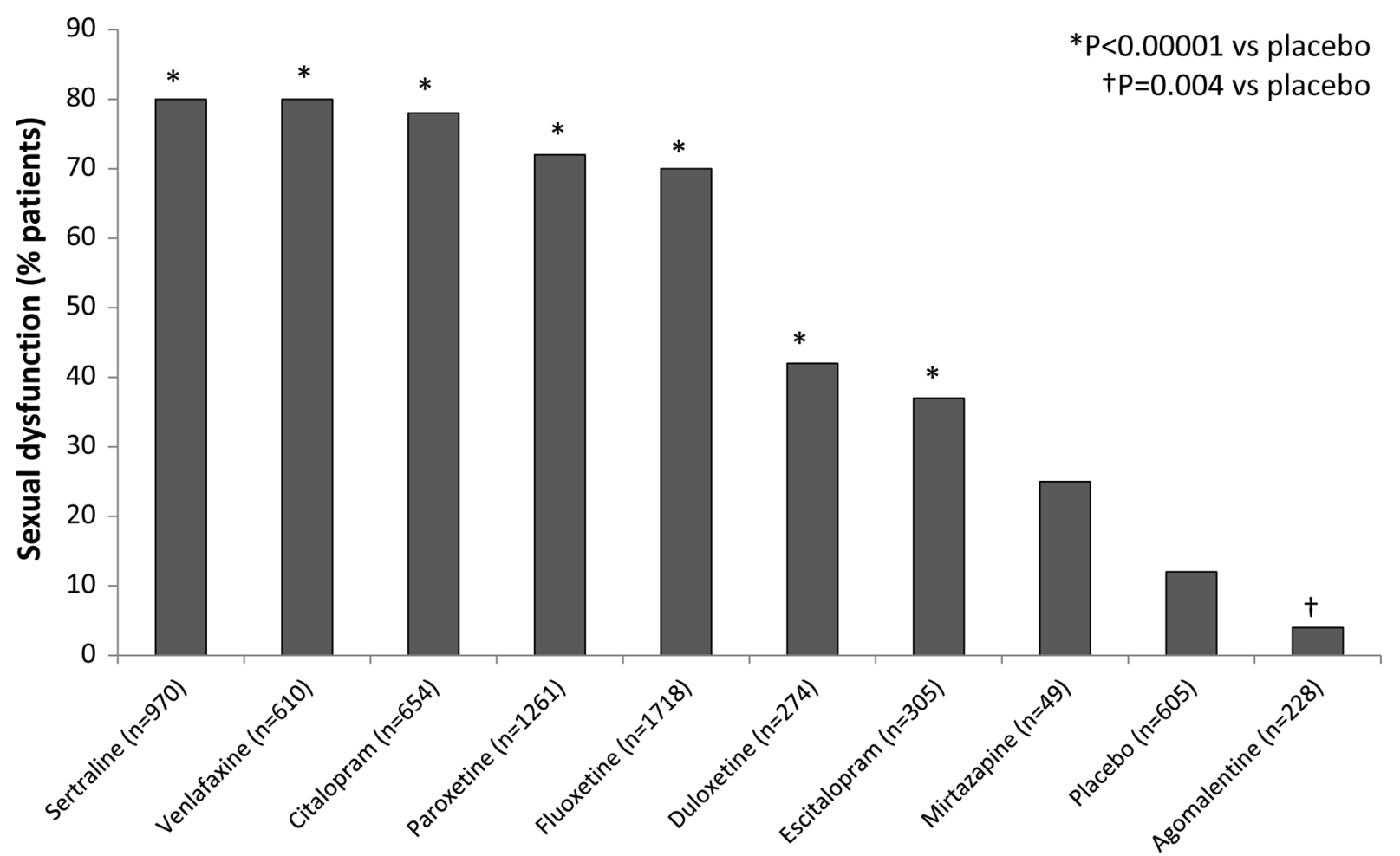

Fig. 3 The incidence of sexual dysfunction at week 8 of treatment with antidepressants in patients with depression. Reproduced with permission from Serretti and Chiesa [37]

Table 5 Modules included in the deprexis ${ }^{\circledR}$ online program

Introduction

Positive cognitive restructuring

Behavioural activation exercises

Relaxation techniques

Mindfulness and self-acceptance

Learning social skills for achieving good relationships

Problem solving

Processing childhood and youth memories

Dream analysis

Positive psychology

Summary

status. Others may be interested in using the tool but are concerned that this will reduce their direct interaction with the treating physician. Therefore, physicians need to identify any underlying patient concerns with the use of online interventions and clearly inform patients that online CBT is an additional tool in the management of their depression and not the cornerstone of therapy. In order to be comfortable with its use, patients should understand that using online CBT will not affect their physician visits or healthcare interactions in any way during treatment. Patients should be motivated to use online CBT at each visit. Similarly, physicians and therapists need to understand that the software is an additional tool in patient management and should not replace direct interaction with patients during appointments. This is particularly important for patients at risk of social isolation, who require regular opportunities to engage with others face to face and discuss their feelings [52].

The deprexis ${ }^{\circledR}$ program is time-limited (3 months), and patients should be advised to start using it immediately after registration to obtain maximum benefit. A nurse who is 
familiar with the online CBT program can give patients an overview of the software before they begin using it. Patients should understand that the software is anonymous, discreet and safe, and at the same time is intuitive, flexible and entertaining. The program identifies unwanted habits and thinking patterns in patients and teaches them to understand themselves better.

Using deprexis ${ }^{\circledR}$ can also be beneficial for treating physicians, because it can explain exercises and depression management methods to patients in their own time, thereby saving time during appointments. The physician or therapist can use the additional face-to-face time during sessions to work on the patient's individual issues in a more focused way.

The following case reports illustrate how deprexis ${ }^{\circledR}$ can benefit both patients and therapists, if used correctly.

\section{Case Report 1}

A middle-aged public servant presented for treatment after being made redundant. The patient was unmarried and had few interpersonal relationships except with a beloved pet. The patient had a rationalising personality and needed a lot of comforting during sessions, but never really opened up about the issues they were facing. Although reluctant to use deprexis ${ }^{\circledR}$ initially, the patient began to use the program and bring printouts of specific exercises and modules to the therapy sessions. This led to greater sharing between the patient and therapist about the lack of appreciation the patient had experienced in life and the need for intimacy and relationships. Effective therapy was able to be initiated once the patient's needs were known.

\section{Case Report 2}

A 38-year-old woman with a demanding job requiring her to work up to $12 \mathrm{~h}$ /day reported to the clinic in significant distress. She had anorexia and a history of using performance-enhancing drugs. The patient decided to quit her job and work on her relationships instead. Subsequently, she became pregnant and chose not to take any medication. She was put on bed rest because of pregnancy complications, and the lack of work and activity in her life made her feel worthless. She was introduced to deprexis ${ }^{\circledR}$ at one of her sessions and was convinced to use it as a support option. She chose the detailed version of the program with audio and video options. Using deprexis ${ }^{\circledR}$ made her feel comforted and cared for; after multiple sessions, she recovered well and was mentally healthy at the last visit.

\section{CONCLUSION}

We are in a new era of depression management, in which a broad range of medications are available. Therefore, it is now possible to choose medications in a rational and thoughtful way based on patient history, family history, patient symptoms, response to treatment and attitudes/ perceptions of the available interventions. In addition, pharmacotherapy can be supplemented by psychotherapeutic interventions, some of which can be accessed online. Like every other available intervention, online CBT has both positive and negative sides. However, it has great promise as an adjunct to therapy in the management of depression, particularly for patients who may not be able to access direct interaction with a psychotherapist because of the severity of their condition, their geographic location or socioeconomic situation. Regardless of the nature of the intervention, obtaining information about a patient's own needs and concerns will help to individualise treatment and foster a collaborative therapeutic relationship between patient and health provider that is likely to maximise the chances of adherence and recovery.

\section{DISCUSSION (Q AND A)}

\section{Q: Should We Consider Recovery Instead of Remission in Patients with Depression?}

Ellen Frank: As observed in clinical practice, patients with depression generally tend to have recurrent episodes, making it a lifelong illness. Effective treatment strategies can increase the 
duration of their remission periods, and in some patients prolonged remission periods may be termed recovery. Recovery may also be used to describe patients who experience a single episode of depression that remits and does not recur. However, it is important to clearly differentiate between recovery and remission: recovery generally suggests response to an intervention (pharmacotherapy, psychotherapy, online CBT, etc.) causing a substantial reduction in symptoms and suffering of the patient, while remission indicates a temporary phase of reduced severity of symptoms. Patients who persist with treatment are more likely to achieve remission and recovery.

David Castle: Currently there is a lack of data on the response of patients to available treatments for depression (using different available scales) indicating remission. It is important to understand that recovery means return to normal functioning and embracing life again. Recovery takes time and cannot be achieved if the patient is highly symptomatic. The first step therefore is to work towards remission, which can help achieve recovery in the long run.

K. Demyttenaere: 'Remission' and 'recovery' have different meanings. Remission means fading away of the negative while recovery means also getting back to positive. Patients with depression have a lifetime memory of it, and some patients may not return to their normal state even with the best therapy. It has been observed (using different scales) that although negative mood normalises in these patients during remission, their positive mood is dramatically below what is considered normal, and these patients felt positive mood only when their scores reached zero. Overall, the longer a patient is depressed, the higher the chances are that he/she will be diagnosed with a personality disorder.

Ellen Frank: It should however be remembered that the patient's scores for positive mood before they had their first depression episode are never available, so data on post-treatment scores need to be interpreted with caution. A study in women with recurrent depression who were tracked for personality disorders for 2 years after remission found that the personality characteristics became less obvious with time and met fewer of the personality disorder characteristics defined by Diagnostic and Statistical Manual of Mental Disorders (DSM).

Q: What is the Effect of Internet-Based Interventions on the Relationship Between the Patients and Their Treating Psychotherapist? Does the Response Depend on Patient Personality?

E. Cindik-Herbrüggen: Based on clinical experience, some patients respond positively to online interventions, which improves the doctor-patient interaction. Others may be more reluctant to use online CBT for multiple reasons; however, the effect of this attitude on the degree of sharing information during interaction with the psychotherapist needs to be studied.

Ellen Frank: The effect of internet-based interventions on the doctor-patient interaction depends on the nature and type of intervention and on how it is presented to the patient. It can have a negative effect if the psychotherapist has a short one-to-one session with the patient and instead asks them to look at the online program at home. On the other hand, using online CBT with standard-length individual sessions and pharmacotherapy may act synergistically and show a positive effect. Since this technologybased technique is new, it may be confusing for some patients, and reinforcing its importance during each session may be useful. Depression is a continuous process and it is often very difficult to fully understand a patient's requirements during weekly/fortnightly 20-min sessions. Online interventions can help the psychotherapist understand what happens with the patient between sessions and support the patient when their therapist is not available. Clinicians who use the online software should be well versed in them, so that they can address any doubts the patient may have.

David Castle: The key to successful use of the online interventions is to understand the available options and work synergistically with the patients. Both psychotherapists and nurses play a key role in making patients comfortable with the use of online CBT to achieve maximum benefit. 
Q: What is the Best Way of Managing Patients with Emotional Blunting When on SSRis? Is Switching to Another Drug a Good Option or Will Treatment with Combination Drugs Be Beneficial?

David Castle: As previously discussed, there are no pre-set protocols for the management of patients with depression. The general approach in clinical practice is to first identify the severity of depression by interacting with the patient. The patient's history, their symptoms, current medications used and any previous use of medications provide enough information to guide the choice of treatment. Patients with a history of severe depression may be treated with a combination of drugs and then slowly weaned off the SSRI to avoid unwanted effects. In cases where relapse is less likely, treatment may be directly switched to other drugs. In rare cases, patients may actually like emotional blunting and not want to switch to other drugs.

Q: Can Internet-Based Interventions Have a Negative Effect on Humanity in the Long Run?

E. Cindik-Herbrüggen: The best way to manage patients with depression is to have more resources for their diagnosis and treatment. Although there is currently more awareness about reducing screen time in both children and adults, it is important to understand that online CBT is only a method of understanding the patient better and is not intended to completely replace therapy.

\section{ACKNOWLEDGEMENTS}

Funding. This symposium, presented on the 7th of October 2018 as part of the 31st European College of Neuropsychopharmacology (ECNP) Congress in Barcelona, Spain, was sponsored by Servier. This supplement, alongside the journal's Rapid Service fee and Open Access fee was funded by Servier. All authors had full access to the content reviewed in this manuscript and take complete responsibility for the integrity and accuracy of this manuscript.

Medical Writing Assistance. The authors thank Nishad Parkar, PhD, of Springer
Healthcare Communications for writing the first draft of this review supplement. This medical writing assistance was funded by Servier, France.

Authorship. All named authors meet the International Committee of Medical Journal Editors (ICMJE) criteria for authorship of this article, take responsibility for the integrity of the work as a whole, and have given their approval for this version to be published.

Disclosures. Agomelatine was developed and is marketed by Servier for the treatment of major depressive episodes in adult patients. Koen Demyttenaere has received honoraria from Boehringer-Ingelheim, Johnson \& Johnson, Livanova, Lundbeck, Recordati and Servier. Ellen Frank has received honoraria from Servier and Pfizer, equity from Psychiatric Assessments, Inc. and HealthRhythms, Inc., is an advisory board member for Servier and a consultant at Sage Therapeutics. David Castle has received research grants from Eli Lilly, Janssen Cilag, Roche, Allergen, Bristol-Myers Squibb, Pfizer, Lundbeck, Astra Zeneca and Hospira. David Castle has also received travel support and honoraria for consultancy and talks from Eli Lilly, Bristol-Myers Squibb, Astra Zeneca, Lundbeck, Janssen Cilag, Pfizer, Organon, Sanofi-Aventis, Wyeth, Hospira and Servier, and is a current advisory board member for Lundbeck, Pfizer, Shire, Servier and LivaNova. He does not knowingly hold stocks or shares in any pharmaceutical company. Elif Cindik-Herbrüggen has received travel grants or honorarium for her expertise as speaker from Biogen, Genzyme, Novartis, Janssen Cilag, Roche, Servier and Berlin-Chemie. She does not hold stocks or shares in any pharmaceutical company.

Compliance with Ethics Guidelines. This article is based on previously conducted studies and does not contain any studies with human participants or animals performed by any of the authors.

Data Availability. Data sharing is not applicable to this article as no datasets were generated or analysed during the current study. 
Open Access. This article is distributed under the terms of the Creative Commons Attribution-NonCommercial 4.0 International License (http://creativecommons.org/licenses/ by-nc/4.0/), which permits any noncommercial use, distribution, and reproduction in any medium, provided you give appropriate credit to the original author(s) and the source, provide a link to the Creative Commons license, and indicate if changes were made.

\section{REFERENCES}

1. Lingam R, Scott J. Treatment non-adherence in affective disorders. Acta Psychiatr Scand. 2002;105(3):164-72.

2. DiMatteo MR, Lepper HS, Croghan TW. Depression is a risk factor for noncompliance with medical treatment: meta-analysis of the effects of anxiety and depression on patient adherence. Arch Intern Med. 2000;160(14):2101-7.

3. Jung WY, Jang SH, Kim SG, et al. Times to discontinue antidepressants over 6 months in patients with major depressive disorder. Psychiatry Investig. 2016;13(4):440-6.

4. Lawrenson RA, Tyrer F, Newson RB, Farmer RD. The treatment of depression in UK general practice: selective serotonin reuptake inhibitors and tricyclic antidepressants compared. J Affect Disord. 2000;59(2):149-57.

5. Frank E, Kupfer DJ, Siegel LR. Alliance not compliance: a philosophy of outpatient care. J Clin Psychiatry. 1995;56(Suppl. 1):11-7.

6. Bull SA, Hu XH, Hunkeler EM, et al. Discontinuation of use and switching of antidepressants: influence of patient-physician communication. JAMA. 2002;288(11):1403-9.

7. Jackson JL, Chamberlin J, Kroenke K. Predictors of patient satisfaction. Soc Sci Med. 2001;52(4): 609-20.

8. Zimmerman M, McGlinchey JB. Why don't psychiatrists use scales to measure outcome when treating depressed patients? J Clin Psychiatry. 2008;69(12):1916-9.

9. Hamilton $\mathrm{M}$. The role of rating scales in psychiatry. Psychol Med. 1976;6(3):347.
10. Glattacker M, Heyduck K, Meffert C, Jakob T. Illness beliefs, treatment beliefs and information needs as starting points for patient information: the evaluation of an intervention for patients with depression. J Clin Psychol Med Settings. 2018;25(3):316-33.

11. Acosta F, Rodriguez L, Cabrera B. Beliefs about depression and its treatments: associated variables and the influence of beliefs on adherence to treatment. Rev Psiquiatr Salud Ment. 2013;6(2):86-92.

12. Rosenblat JD, Simon GE, Sachs GS, et al. Treatment effectiveness and tolerability outcomes that are most important to individuals with bipolar and unipolar depression. J Affect Disord. 2019;243:116-20.

13. Cowan K, Oliver S. The James Lind Alliance guidebook, version 5. UK: National Institute for Health Research; 2013. http://www.jlaguidebook.org/.

14. James Lind Alliance. Depression: asking the right questions; 2016. http://www.jla.nihr.ac.uk/prioritysetting-partnerships/depression/downloads/Depres sion-PSP-final-report.pdf.

15. Charpeaud T, Samalin L, Garnier M, et al. Choisir mon traitement avec mon médecin: Guide d'aide à la décision médicale partagée destiné aux personnes souffrant d'une dépression (in preparation). https://www.chu-clermontferrand.fr/Internet/Docum ents/Actu/2018/Guide\%20DMP\%20depression\%202 018\%20copyright.pdf

16. Frank E, Kupfer DJ, Perel JM, et al. Three-year outcomes for maintenance therapies in recurrent depression. Arch Gen Psychiatry. 1990;47(12):1093-9.

17. Frank E, Kupfer DJ, Thase ME, et al. Two-year outcomes for interpersonal and social rhythm therapy in individuals with bipolar I disorder. Arch Gen Psychiatry. 2005;62(9):996-1004.

18. Reynolds CF 3rd, Frank E, Perel JM, et al. Nortriptyline and interpersonal psychotherapy as maintenance therapies for recurrent major depression: a randomized controlled trial in patients older than 59 years. JAMA. 1999;281(1):39-45.

19. Swartz HA, Frank E, Zuckoff A, et al. Brief interpersonal psychotherapy for depressed mothers whose children are receiving psychiatric treatment. Am J Psychiatry. 2008;165(9):1155-62.

20. Thomson L, Barker M, Kaylor-Hughes C, et al. How is a specialist depression service effective for persistent moderate to severe depressive disorder?: a qualitative study of service user experience. BMC Psychiatry. 2018;18(1):194. 
21. Howland RH. Sequenced Treatment Alternatives to Relieve Depression (STAR*D). Part 2: study outcomes. J Psychosoc Nurs Ment Health Serv. 2008;46(10):21-4.

22. Howland RH. Sequenced Treatment Alternatives to Relieve Depression (STAR*D). Part 1: study design. J Psychosoc Nurs Ment Health Serv. 2008;46(9):21-4.

23. Angst J, Azorin JM, Bowden CL, et al. Prevalence and characteristics of undiagnosed bipolar disorders in patients with a major depressive episode: the BRIDGE study. Arch Gen Psychiatry. 2011;68(8): 791-8.

24. Zubenko GS, Sommer BR, Cohen BM. On the marketing and use of pharmacogenetic tests for psychiatric treatment. JAMA Psychiatry. 2018;75(8): 769-70.

25. Dunlop BW, Rajendra JK, Craighead WE, et al. Functional connectivity of the subcallosal cingulate cortex and differential outcomes to treatment with cognitive-behavioral therapy or antidepressant medication for major depressive disorder. Am J Psychiatry. 2017;174(6):533-45.

26. Rutherford BR, Wall MM, Brown PJ, et al. Patient expectancy as a mediator of placebo effects in antidepressant clinical trials. Am J Psychiatry. 2017;174(2):135-42.

27. Linden M. Placebo: unsolved problems for science, and simple conclusions for clinical practice. Am J Psychiatry. 2017;174(2):91-2.

28. Dunlop BW, Kelley ME, Aponte-Rivera V, et al. Effects of patient preferences on outcomes in the predictors of remission in depression to individual and combined treatments (PReDICT) study. Am J Psychiatry. 2017;174(6):546-56.

29. Hamann J, Leucht S, Kissling W. Shared decision making in psychiatry. Acta Psychiatr Scand. 2003;107(6):403-9.

30. Goodwin GM, Price J, De Bodinat C, Laredo J. Emotional blunting with antidepressant treatments: a survey among depressed patients. J Affect Disord. 2017;221:31-5.

31. Price J, Goodwin GM. Emotional blunting or reduced reactivity following remission of major depression. Medicographia. 2009;31:152-6.

32. Price J, Cole V, Goodwin GM. Emotional side-effects of selective serotonin reuptake inhibitors: qualitative study. $\mathrm{Br} \mathrm{J}$ Psychiatry. 2009;195(3):211-7.
33. Corruble E, Belaidi C, Goodwin G. P02-24-Agomelatine versus escitalopram in major depressive disorders: a randomized double-blind, long term study focusing on sleep satisfaction and emotional blunting. Eur Psychiatry. 2011;26:619.

34. Martinotti G, Sepede G, Gambi F, et al. Agomelatine versus venlafaxine XR in the treatment of anhedonia in major depressive disorder: a pilot study. J Clin Psychopharmacol. 2012;32(4):487-91.

35. Castle D, Hopwood M, Crawford A. Raising the profile of affective blunting in general practice. Aust N Z J Psychiatry. 2015;49(Suppl. 1):106.

36. Demyttenaere K, Mortier P, Kiekens G, Bruffaerts R. Is there enough "interest in and pleasure in" the concept of depression? The development of the Leuven Affect and Pleasure Scale (LAPS). CNS Spectr. 2019:24:265-74.

37. Serretti A, Chiesa A. Treatment-emergent sexual dysfunction related to antidepressants: a metaanalysis. J Clin Psychopharmacol. 2009;29(3):259-66.

38. Harter M, Klesse C, Bermejo I, Schneider F, Berger M. Unipolar depression: diagnostic and therapeutic recommendations from the current S3/National Clinical Practice Guideline. Dtsch Arztebl Int. 2010;107(40):700-8.

39. Ein Jahr nach der Reform der PsychotherapieRichtlinie. Wartezeiten 2018. Berline: Bundes Psychotherapeutic Kammer; 2018.

40. Stoppe G. Es gibt keine Gesundheit ohne psychische Gesundheit. Deutsches Ärzteblatt. 2013;110:A543-6.

41. Meyer B, Berger T, Caspar F, et al. Effectiveness of a novel integrative online treatment for depression (Deprexis): randomized controlled trial. J Med Internet Res. 2009;11(2):e15.

42. Beevers CG, Pearson R, Hoffman JS, et al. Effectiveness of an internet intervention (Deprexis) for depression in a united states adult sample: a parallel-group pragmatic randomized controlled trial. J Consult Clin Psychol. 2017;85(4):367-80.

43. Berger T, Hammerli K, Gubser N, Andersson G, Caspar F. Internet-based treatment of depression: a randomized controlled trial comparing guided with unguided self-help. Cogn Behav Ther. 2011;40(4):251-66.

44. Berger T, Krieger T, Sude K, Meyer B, Maercker A. Evaluating an e-mental health program ("deprexis") as adjunctive treatment tool in psychotherapy for depression: results of a pragmatic randomized controlled trial. J Affect Disord. 2018;227:455-62. 
45. Klein JP, Berger T, Schroder J, et al. Effects of a psychological internet intervention in the treatment of mild to moderate depressive symptoms: results of the EVIDENT study, a randomized controlled trial. Psychother Psychosom. 2016;85(4):218-28.

46. Meyer B, Bierbrodt J, Schröder J, et al. Effects of an internet intervention (Deprexis) on severe depression symptoms: randomized controlled trial. Internet Interv. 2015;2(1):48-59.

47. Moritz S, Schilling L, Hauschildt M, Schroder J, Treszl A. A randomized controlled trial of internetbased therapy in depression. Behav Res Ther. 2012;50(7-8):513-21.

48. Zwerenz R, Becker J, Knickenberg RJ, et al. Online self-help as an add-on to inpatient psychotherapy: efficacy of a new blended treatment approach. Psychother Psychosom. 2017;86(6):341-50.
49. Meyer B, Bültmann O, Jacob G. Efficacy of deprexis, an internet-based depression treatment: a metaanalysis of ten randomized controlled trials. In: 31st Congress of the European College of Neuropyschopharmacology, 6-9 Oct, Barcelona, Spain; 2018.

50. Twomey C, O’Reilly G, Meyer B. Effectiveness of an individually-tailored computerised CBT programme (Deprexis) for depression: a meta-analysis. Psychiatry Res. 2017;256:371-7.

51. Gräfe V, Greiner W. Internet based treatment of depressive symptoms-a health economic evaluation of costs and benefits. Value Health. 2017;20(9):A714.

52. Walsh S, Kaselionyte J, Taylor SJC, Priebe S. What might affect acceptability of online positive psychology interventions for depression: a qualitative study on patient expectations'. BMC Psychiatry. 2018;18(1):240. 